Results Hospice healthcare professionals highlighted challenges when providing palliative care to individuals from minority ethnic communities, particularly language barriers. Minority ethnic communities in South Wales appeared uninformed about local palliative care services and the keyworker implemented a range of initiatives to raise awareness and widen hospice referral routes. Additionally, the keyworker supported healthcare professionals when caring for people from minority ethnic communities, for example meeting religious needs at the end-of-life.

Looking ahead, hospice staff are keen to increase their understanding of different ethnic groups and cultures, while the keyworker hopes to work with community healthcare teams to improve referral rates to the hospice.

Conclusions and application The evaluation has demonstrated the progress made by the keyworker to engage minority ethnic communities and support hospice staff to holistically care for people from different ethnic groups.

The keyworker role appears, at this early stage in the project, to be an excellent way of engaging minority ethnic communities and improving access to palliative care services.

\section{P166 WHEN ENGLISH ISN'T YOUR FIRST LANGUAGE: SUPPORTING DEAF PEOPLE IN END OF LIFE CARE}

${ }^{1}$ Melanie Hodson, ${ }^{2} \mathrm{Jill}$ Ede, ${ }^{3}$ Rowena Dean, ${ }^{4}$ Rebecca Edwards. ${ }^{1}$ Help the Hospices, London, UK, ${ }^{2}$ Info to Go Ltd, Cambridge, UK, ${ }^{3}$ SignHealth, Beaconsfield, UK, ${ }^{4}$ Remark!, London, UK

\subsection{6/bmjspcare-2013-000591.188}

Identified as a significant public health issue, hearing loss affects more than 10 million people in the UK. This figure is expected to rise to 14.5 million by 2031 [1] and the WHO anticipates that adult onset hearing loss will be amongst the top ten health problems" in the UK by 2030 [2]. Approximately 50,000 people use British Sign Language (BSL) as their first or preferred language [3].

It is thought that of those dying each year in England and Wales, more than 70,000 will be deaf or hard of hearing and it is reported that there is 'little deaf awareness within the world of palliative care' [4].

Low deaf awareness amongst health staff and insufficient communication support means that people with hearing loss can find health services difficult and frustrating to use and family members may be inappropriately used as interpreters.

Access to end of life care can be impeded by communication difficulties and low health literacy. Research suggests that people who are deaf may have limited understanding of their choices for end of life care [5]. Pain and symptom management can be key issues [6] and a literature review indicates that there is little information available in BSL to help people find out about hospice care.

We discuss barriers to communication, impacts of a lack of deaf awareness, outline ways in which hospice care professionals can support people who are deaf and present a new BSL hospice care information resource.

\section{P167 AN EXPLORATION OF END OF LIFE CARE NEEDS OF PEOPLE WITH INTELLECTUAL DISABILITIES: DEVELOPING A MODEL OF SERVICE DELIVERY}

${ }^{1}$ Kumaresan Cithambaram, ${ }^{2}$ Mel Duffy, ${ }^{3}$ Eileen Courtney. ${ }^{1}$ Dublin City University, Dublin, Ireland, Republic of, ${ }^{2}$ Dublin City Uiversity, Ieland, Rpublic of, ${ }^{3}$ Dblin Cty University, Ireland, Republic of

10.1136/bmjspcare-2013-000591.189
Background People with intellectual disabilities are living longer and suffering from the same life limiting illness as general population. The changing needs and care demands of this group force service providers and professionals to better equip themselves to respond and attend to the specific health needs of this group. In the caring process service providers often forget to hear, listen to and include the voices of this vulnerable population which can lead to people with intellectual disabilities receiving inconsistent and inappropriate end-of-life care.

Aim To explore the end-of-life care needs of people with intellectual disabilities and develop a model of service delivery.

Objectives 1. To explore attitudes, perceptions and preferences of people with intellectual disabilities about end-of-life care. 2 . To explore perceptions and experiences of family members of people with intellectual disabilities about end-of-life care needs of people with intellectual disabilities. 3. To develop a model of service delivery for people with intellectual disabilities at the end of life.

Methodology The study was conducted in an intellectual disability service that provide residential and community service to adult with intellectual disabilities. Constructivist version of Grounded Theory Methodology was adopted. Ethical permission was obtained from the academic institution and service to carry out the study. All consenting participants (17) were interviewed between 45-60 minutes. Data were analysed using Constant comparative method.

Findings The data revealed how the participants wanted to be cared at the time of dying and after death. The participants' main concerns are revealed from the data are Holism and Familiarism which explain the care needs at the time of dying and Respectivism which explains the care requirement after death. A core category of Continum and Ultimatum was constructed.

Conclusion and Implication The findings from this study will help to develop a model of service to meet the needs of people with intellectual disabilities at the end of life.

\section{P168 LEARNING DISABILITIES AND PALLIATIVE CARE: BUILDING BRIDGES - SUPPORTING CARE}

Liz Smith, Allison O'Donnell. The Prince and Princess of Wales Hospice, Glasgow, Scotland

\subsection{6/bmjspcare-2013-000591.190}

Palliative care for people with learning disabilities (PWLD) is often complex but it is vital that these challenges are faced. It is also important to recognise that life limiting illness is more prevalent in the learning disability population and that PWLD die on average 25 years younger than the general population $^{1-2}$. Increasing numbers of PWLD are living at both ends of the age spectrum with co- morbid conditions and complex health needs and there is a growing need for palliative care provision.

This project recognises the need for education and the development of robust partnerships which lead to collaborative working, to ensure that PWLD will experience equity in accessing palliative care services.

The aim of the project, funded through Help the Hospices and the Scottish Government, is to bring the two specialities of Palliative Care and Learning Disabilities with Greater Glasgow \& Clyde together using a practice development approach. This will provide support to staff in the provision of best quality care for people with Learning Disabilities who have palliative care needs. 